\title{
First steps in the development of the Multi Ion Reflection Apparatus for Collinear Laser Spectroscopy
}

\author{
S. Sels ${ }^{\mathrm{a}, *}$, P. Fischer ${ }^{\mathrm{b}}$, H. Heylen ${ }^{\mathrm{a}}$, V. Lagaki ${ }^{\mathrm{a}, \mathrm{b}}$, S. Lechner ${ }^{\mathrm{a}, \mathrm{e}}$, F.M. Maier $^{\mathrm{a}, \mathrm{c}}$, P. Plattner $^{\mathrm{a}, \mathrm{f}}$, M. Rosenbusch $^{\mathrm{b}, 2}$, \\ F. Wienholtz ${ }^{\mathrm{a}, \mathrm{b}}$, R.N. Wolf ${ }^{\mathrm{b}, 1}$, W. Nörtershäuser ${ }^{\mathrm{d}}$, L. Schweikhard ${ }^{\mathrm{b}}$, S. Malbrunot-Ettenauer ${ }^{\mathrm{a}}$ \\ ${ }^{a}$ ISOLDE, CERN Experimental Physics Department, CH-1211 Geneve 23, Switzerland \\ ${ }^{b}$ Institut für Physik, Universität Greifswald, 17487 Greifswald, Germany \\ ${ }^{c}$ Johannes Kepler University Linz, Altenbergerstrasse 69, 4040 Linz, Austria \\ ${ }^{d}$ Technische Universität Darmstadt, Karolinenpl. 5, 64289 Darmstadt, Germany \\ ${ }^{e}$ Technische Universität Wien, Karlsplatz 13, 1040 Wien, Austria \\ ${ }^{f}$ Universität Innsbruck, Innrain 52, 6020 Innsbruck, Austria
}

\begin{abstract}
Collinear laser spectroscopy (CLS) has been combined with the multi-reflection time-of-flight (MR-ToF) technique. To this end, a photodetection system has been implemented at the drift region of a MR-ToF apparatus and a laser beam has been sent along the path of the ions that are stored between the two ion-optical mirrors. The main goal of the present proof-of-principle $(\mathrm{PoP})$ experiments, is the confirmation of the expected increase in sensitivity compared to conventional fluorescence-based CLS due to the repeated probing of the trapped ion bunches. The novel method will be used for the precise measurement of nuclear ground- and isomeric-state properties of exotic nuclei with low production yields at radioactive ion-beam facilities. A significant sensitivity improvement of CLS is expected, depending on the half-life and mass of the nuclide of interest. The status of the PoP setup and future improvements are discussed.
\end{abstract}

\section{Introduction}

Collinear laser spectroscopy (CLS) is a well-established ${ }_{25}$ technique to measure ground- and isomeric-state properties of radioactive nuclides 1, 2, From the hyperfine split5 ting (HFS) and isotope shift (IS) of optical transitions for different isotopes in an isotopic chain, one can deduce the electromagnetic moments and mean-square charge-radii differences in a nuclear-model-independent way. In conventional, fluorescence-based CLS at radioactive ion-beam (RIB) 10 facilities such as ISOLDE at CERN 3, 4, beams of radioactive isotopes are produced and accelerated to an energy of several tens of keV. These beams of singly-charged ${ }_{35}$ ions or neutralized atoms are collinearly (or anticollinearly) overlapped with a narrow-band, continuous-wave laser that

15 resonantly excites the optical transitions. Usually, the frequency observed by the atoms or ions is Doppler tuned by adjusting the ion-bunch acceleration voltage, while keeping the laser-frequency fixed in the lab frame. Fluorescence photons emitted by the laser-excited ions or atoms 20 passing an optical detection region (ODR) are observed by use of photomultiplier tubes. After passing the ODR only once, with an interaction time of only a few microseconds ${ }_{45}$

\footnotetext{
* Corresponding author

Email address: simon.sels@cern.ch (S. Sels)

${ }^{1}$ Current address: ARC Centre for Engineered Quantum Systems, The University of Sydney, Australia

${ }^{2}$ Current address: RIKEN Nishina Center for Accelerator-Based Science, Wako, Saitama 351-0198, Japan
}

5], the ion bunch is usually sent to a beam dump. However, ISOL facilities such as ISOLDE deliver radioactive nuclides with half-lives ranging from about a millisecond up to several years or even stable [6. This causes a large mismatch between nuclear lifetime and observation time, which leaves room for improvement of the experimental sensitivity. Such an improvement is crucial if one wishes to extend high-resolution CLS measurements to exotic nuclides with very low production yields that are currently out of reach for conventional CLS.

The development of a Multi Ion Reflection Apparatus for Collinear Laser Spectroscopy (MIRACLS) at CERN aims to increase the sensitivity of conventional CLS by addressing this mismatch between nuclear lifetime and observation time. By reflecting the ion bunches back and forth between the two electrostatic mirrors of a MultiReflection Time-of-Flight (MR-ToF) device [7-16, they can be probed by the laser beam during each revolution. At today's radioactive ion beam facilities, MR-ToF devices are mostly used for mass spectrometry or mass separation. By confining ion bunches for several thousand revolutions, their time-of-flight (ToF) is increased and even ion species with minute mass differences separate over time. MR-ToF devices reach mass-resolving powers $R=\frac{m}{\Delta m}$ of several hundred thousands in less than $30 \mathrm{~ms}[17-19]$.

To date, MR-ToF devices usually work at ion energies of $\approx 1-2 \mathrm{keV}[10,17,19,20$. However, in order to preserve 
the high spectroscopic resolution of conventional CLS, the final MIRALCS setup will operate at typical CLS-energies of $E \geq 30 \mathrm{keV}$. This allows one to approach the natural line widths, as the Doppler line-width of the atomic transition $\delta_{f}$ scales with the ion-beam energy as $\delta_{f} \propto \delta_{E} / \sqrt{E}$, where $\delta_{E}$ represents the ions' energy spread [21]. In the current, first stage of the project, a proof-of-principle ( $\mathrm{PoP}$ ) setup has been assembled by adapting a low-energy MR-ToF device 22-24 for preliminary CLS tests. This PoP setup will be utilized to experimentally demonstrate the MIRACLS concept, further develop the technique, and benchmark simulations 25 that are employed to design the future 30-keV apparatus.

\section{Proof-of-principle setup}

A schematic of the offline PoP setup is shown in Fig. 1 An electron-impact ion source (adapted from [26]), floated to $\approx 250 \mathrm{~V}$, provides a continuous ion beam of stable magnesium. The beam is focused into a helium-filled radio70 frequency quadrupole (RFQ) cooler-buncher, where the ions are cooled and accumulated. After being released from the RFQ, the ion bunch is accelerated by a crownshaped acceleration electrode and pulsed drift tube (PDT) to an energy of $\approx 2 \mathrm{keV}$, before it is deflected onto the

${ }_{75} \mathrm{MR}-\mathrm{ToF}$ axis by a $90^{\circ}$ quadrupole bender. The ions enter the MR-ToF apparatus, where they are decelerated to $\approx 1.3 \mathrm{keV}$ and captured using the in-trap-lift technique [27, 28]. During the time the ion bunches are trapped, they perform multiple revolutions between the two elec-

80 trostatic mirrors of the MR-ToF device. Each mirror consists of 4 cylindrical electrodes to which electric potentials are applied. These are optimized with respect to ion trapping and focusing, while keeping the central drift region of the instrument free of fringe fields [25]. This central

${ }_{85}$ drift section is fully surrounded by a mesh electrode that defines the electrical potential. This mesh electrode has a locally-adjusted wire-spacing which provides an enhanced photon transparency near the ODR. As part of the ODR, an optical lens system [29] and a photomultiplier tube are 90 mounted above the mesh electrode to detect fluorescence photons of laser-excited $\mathrm{Mg}^{+}$ions. The in-trap lift mechanism is also used to eject the ion bunches from the MRToF device 27, 30. The extracted bunches pass a second $90^{\circ}$ quadrupole bender and can be recorded on a multi95 channel plate detector (MCP) for monitoring and beamtuning purposes.

The laser beam for photoexcitation enters the setup ${ }^{135}$ through a quartz, Brewster-angled window and is over100 lapped with the axis of the MR-ToF device. When the laser frequency is in resonance with a Doppler-shifted optical transition of the $1.3-\mathrm{keV}$ ion bunch fluorescence photons are detected in the PMT. By scanning the laser frequency and monitoring the photon signal, the hyperfine-

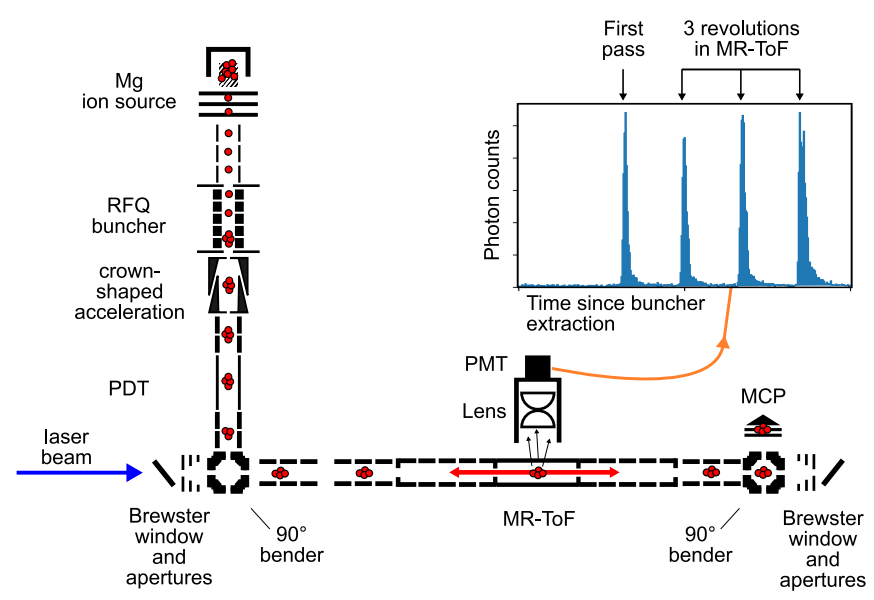

Figure 1: Schematic of the MIRACLS proof-of-principle setup. See text for details.

The improvement with respect to conventional CLS is obvious, as the ion bunches pass the ODR during every revolution in the MR-ToF device, thereby increasing the photon counts and thus the statistical significance of the data with every additional passing.

Stable magnesium ions will be used as an ideal testcase, since the even-even isotopes, ${ }^{24,26} \mathrm{Mg}$, have no hyperfine splitting of the fine-structure levels due to their $I=0$ nuclear spin and the $D_{1}$ and $D_{2}$ transitions at about $280 \mathrm{~nm}$ both form a closed, two-level system. This avoids that, after several revolutions in the MR-ToF device, the population is optically pumped towards a hyperfine level or another fine-structure state which is not probed by the laser and which would therefore result in a decrease in photosignal. Repopulation techniques for more complicated ionic systems will be developed at a later stage of the project.

One of the major advances in CLS on radioactive ions was the introduction of bunched beams to increase the signal-to-noise ratio [31, 32. The reduction is due to the possibility of gating the data acquisition only on the time when the ion bunch passes the ODR. Besides bunching of the ion beam, several other measures are taken in order to further reduce the influence of background in MIRACLS' PoP setup, which, due to its history, was not designed to minimize sources of laser scattering as otherwise imperative for efficient CLS. Hence, a Brewster window and set of three apertures are mounted on either side of the setup as optical ports (see Fig. 1). Together with a stray-light shield painted in photon-absorbing black paint, placed around the ODR, these modifications reduce the laser-induced background by more than an order of magnitude, without noticeably affecting the quality of the vacuum. Indeed, a high vacuum better than $10^{-7} \mathrm{mbar}$ is preferential to reduce the amount of stray-light scattering centers and collisional-induced excitation. Moreover, 
collisions with residual gas particles has been identified motivates a series of UHV upgrades foreseen for the near future, in particular the better separation of the vacuum section of the MR-ToF device from the buffer-gas filled cooler-buncher.

\section{Laser setup}
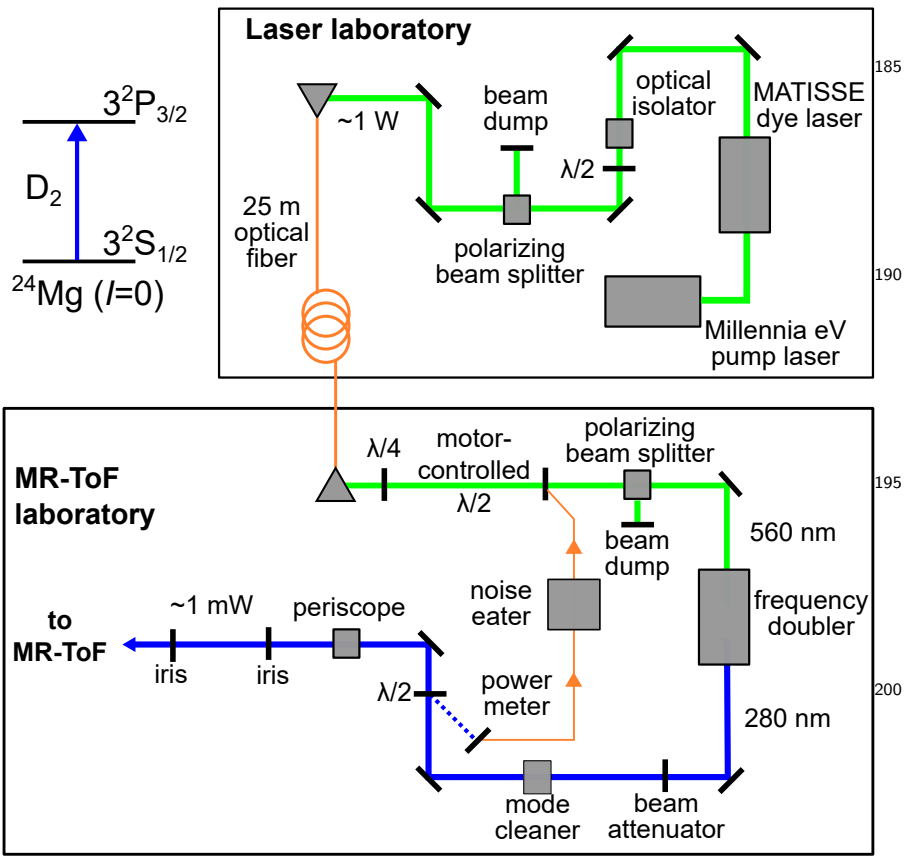

Figure 2: Schematic of the laser setup at MIRACLS PoP experiment. See text for details.

The laser beam at $280 \mathrm{~nm}$, used to resonantly excite the $D_{2}$-like $\left(3^{2} S_{1 / 2} \rightarrow 3^{2} P_{3 / 2}\right)$ transition in magnesium ions, is generated using the setup shown in Fig. 2. A Spectra Physics 20-W Millennia eV laser, produces the 532-nm laser light used to pump a Sirah MATISSE laser operated with Rhodamine 560 dye dissolved in ethylene glycol. The $560-\mathrm{nm}$ output light is coupled into a high-power, largemode-area optical fiber (LMA-15 single-mode $15 \mu \mathrm{m}$ core fiber). Prior to the fiber coupling, a combination of a $\lambda / 2$ plate and a polarizing beam splitter allows for a variable laser-beam intensity during the optimization of the fiber coupling. An optical isolator removes undesirable effects on the dye-laser locking caused by back reflections from the fiber coupler.

The approximately 25-m long optical fiber transports the laser light from the laser laboratory to the MR-ToF lab with a typical efficiency of 50-70\%. The laser light leaving an optical fiber is usually randomly polarized. As unwanted circular polarization would lead to losses (at a second polarizing beam splitter cube further downstream) a quarter wave plate is installed behind the fiber to remove any undesired portion of the polarization. Then, the second-harmonic at $280 \mathrm{~nm}$ is generated in a BBO crystal by use of a Wavetrain frequency doubler. A mode cleaner with a $15-\mu \mathrm{m}$ pinhole is used to remove side fringes originating from the second-harmonic generating process and to obtain a pure Gaussian TEM00 beam profile. This reduces significantly stray-light caused by spatially more extended mode contributions. The laser power introduced into the MR-ToF vacuum setup can be adjusted with a beam attenuator in front of the mode cleaner. The power level is additionally stabilized by guiding the back reflection of a $\lambda / 2$-plate onto a power meter. Its output is used as the process variable of a PI feedback-loop that rotates a motor-controlled $\lambda / 2$-plate in front of the frequency doubler (see Fig.2).

Finally, the 2-mm diameter laser beam is guided through a periscope and two irises to the Brewster windows where it enters the vacuum chamber ( see Fig. 1). The polarization is adjusted to minimize reflections from the Brewster windows using the previously mentioned $\lambda / 2$-plate behind the mode cleaner.

To record the hyperfine structure of an isotope, the laser frequency is scanned across the relevant frequency range, while the laser is stabilized to a High-Finesse WSU10 wavelength meter. The wavelength meter is regularly calibrated using a diode laser that is locked to the ${ }^{87} \mathrm{Rb}$ $\left(S_{1 / 2} \rightarrow P_{3 / 2}\right)$ hyperfine transition at approximately $780 \mathrm{~nm}$. The option of scanning the laser frequency rather than scanning the ion-bunch energy is preferred, since the latter would also change the ion trajectories inside the MR-ToF device.

\section{First signals}

To demonstrate that CLS can be performed within the MIRALCS PoP setup, a resonance spectrum of ${ }^{24} \mathrm{Mg}^{+}$ions passing through the ODR once, i.e. in the conventional collinear laser-spectroscopy mode without MR-ToF operation, is shown in Fig. 3 .

SIMION 33 simulations of the ion transport from the RFQ to the MR-ToF device show that Doppler broadening is the main contributing process to the total linewidth of the measured spectrum at energies of $1.3 \mathrm{keV}$ [25] as the natural line width is only $42 \mathrm{MHz} 34$. A total broadening of $\approx 370 \mathrm{MHz}$ is expected, in accordance with the experimental observation of about $400 \mathrm{MHz}$ as depicted in Fig.3. This spectrum was obtained after tuning of parameters for the ion injection into the MR-ToF device and by working in a regime below the space-charge limit. This resonance shows a slight tailing at the low-frequency side. Most probably, this is related to the distribution of angles of the ion trajectories with respect to the optical axis due to the transversal ion-beam emittance. Each angular deviation $\alpha$ contributes to additional photon counts on the lower-energy tail of the observed spectrum due to the Doppler-shifted laser frequency $f$ in the rest frame of the 


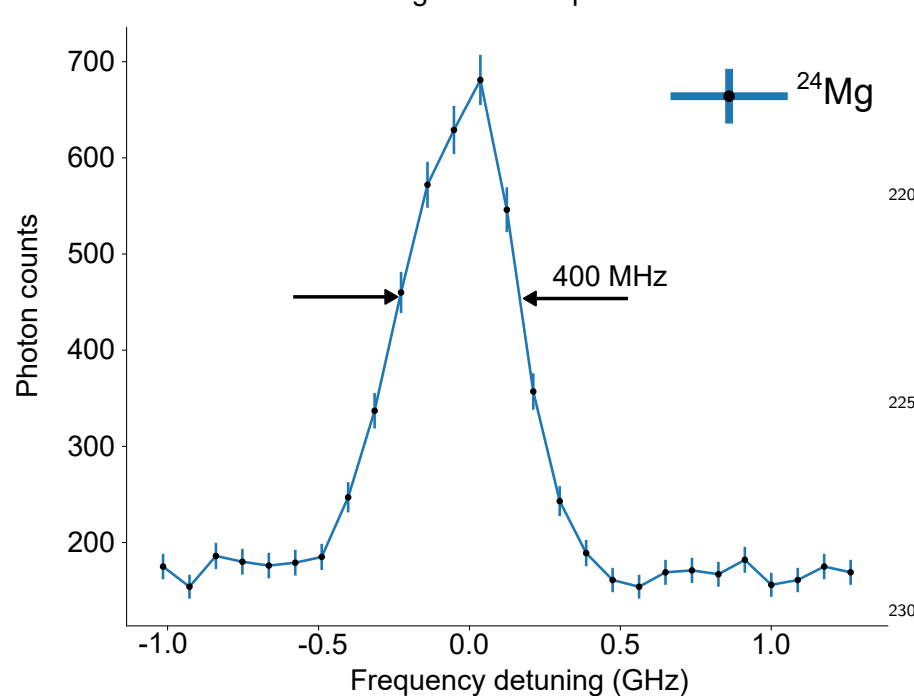

Figure 3: Example of a resonance of ${ }^{24} \mathrm{Mg}^{+}$at a kinetic energy of $\sim 1.55 \mathrm{keV}$ obtained for a single passage through the ODR in the center of the MR-ToF device. The photon counts of 300 ion $^{235}$ bunches have been added for each probe frequency. The data for this spectrum was gated on the passage of the ion bunch as described in section 2 .

ions. This frequency $f$ is described as

$$
f=f_{0} \frac{\sqrt{1-\left(\frac{v}{c}\right)^{2}}}{1-\frac{v_{x}}{c}},
$$

where $c$ represents the speed of light, $v$ the total velocity ${ }^{245}$ of the ions, and $v_{x}=v \cos \alpha$ the velocity component along the MR-ToF axis.

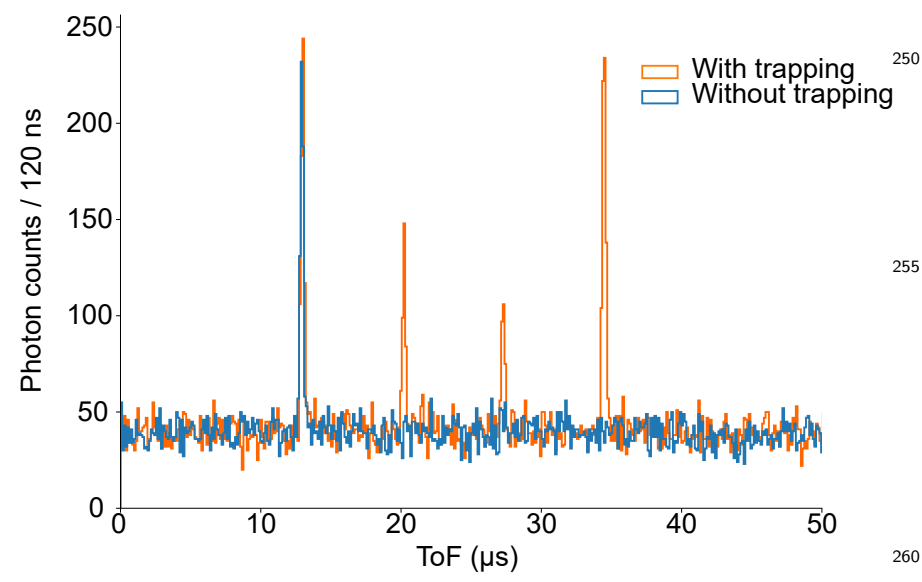

Figure 4: Photomultiplier count rates as a function of time with trapping, i.e. MR-ToF operation (orange) and without (blue), i.e. singlepass mode. The laser frequency is tuned to resonance. This provides a direct comparison between sensitivity in conventional CLS and in the MIRACLS approach. The photon counts of 300 ion bunches have265 been added.

Examples of the resonant fluorescence signal as a function of time are presented in Fig. 4. once for single-pass
215 operation (blue line) as in conventional CLS and once for MR-ToF operation where the ions are trapped for three revolutions (orange line) and thus produce three further fluorescence signals when passing in front of the ODR. When the laser is detuned, the fluorescence signals disappear and only the background level is left (not shown). Hence, the multiple passages of the ions through the ODR as enabled by the MIRACLS approach substantially increase the number of photon counts and thus the statistical significance of the collected data.

We note in passing that the fluorescence signal fluctuates during the first revolutions in the MR-ToF apparatus. While this phenomenon is out of the scope of the present study we have observed a stabilization of the signal at higher revolution numbers. The signal-height fluctuations during the first revolutions after ion injection depend very strongly on the injection parameters and similar observations are known from the ion signal as a function of revolution number at other MR-ToF devices 35. From the recurrence of the peak intensity it can be deduced that the ion beam is not lost, but occasionally merely on a trajectory not overlapping with the laser beam. When the ion bunch is tuned into the MR-ToF device differently, this effect can be reduced, as shown for instance in the inset of than just the signal height, the evolution and control of other signal parameter are being studied as a function of revolution number in the $\mathrm{PoP}$ setup (e.g. signal-width) 36 .

As conventional MR-ToF operation provides ion trapping for a few thousand revolutions [17, a significant increase in experimental sensitivity is anticipated for MIRACLS. It will make it possible to isolate the CLS signal even for exotic nuclides with low production yields, for which a conventional, i.e. single-pass, signal would be buried in the background. This is simulated in Fig.5 for a case where single passage through the ODR cannot produce an identifiable signal (top), but where a signal-to-noise ratio of about 1 is reached after 100 revolutions (middle). Consequently, a clear signal with good statistics is obtained after about 1000 revolutions (bottom).

\section{Outlook}

The possibilities offered by the MIRACLS technique will be further explored in the future, first in the offline PoP setup described above, later in the $30-\mathrm{keV}$ device that is currently being designed. Early anticipated advances include trapping of the ion bunches for several thousands of revolutions to increase the improvement factor of MIRACLS. The development of the centroid, spectral linewidth and signal strength with the number of revolution periods will be studied for these extended trapping times. As a first test case, the well-known isotope shifts of stable magnesium isotopes will be measured and compared to the literature values to investigate the accuracy that can 


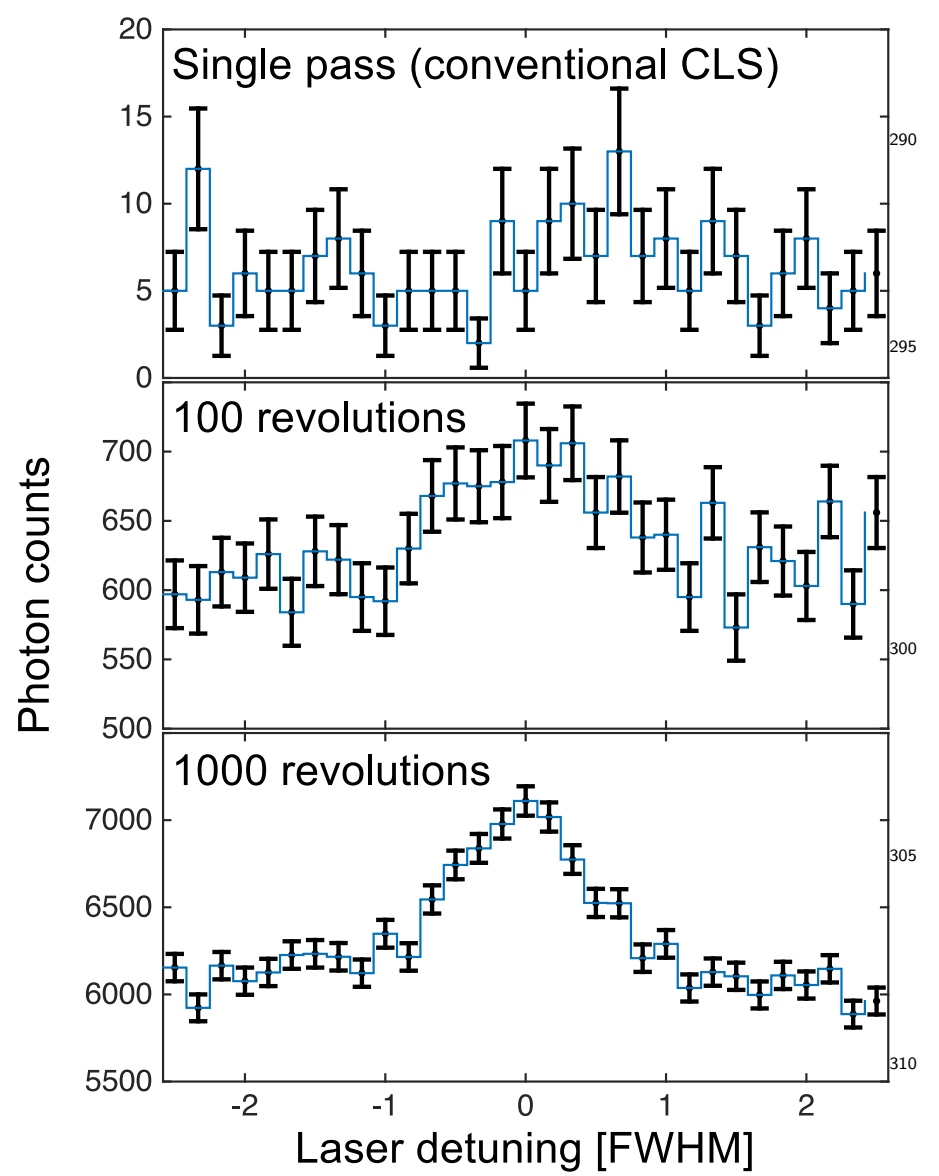

Figure 5: Simulated photon counts for an ion bunch passing the ODR only once (top) and for 100 and 1000 revolutions in the MR-ToF device (middle and bottom, respectively). Ion losses from radioactive decay or trapping in the MR-ToF device are not included in this ${ }_{315}$ representation.

be reached with the PoP apparatus of MIRACLS. It will later be extended to other elements. In its first stage, theз20 technique would be most useful for closed, two-level transitions. In order to expand towards more general cases, research on the redistribution of excited states of the ion ensemble using additional lasers will be performed. An-325 other advance could be the addition of a second laser to perform simultaneous collinear and anticollinear measurements as was recently demonstrated for beryllium isotopes 37, 38. and applied at even higher accuracy for barium ${ }^{330}$ isotopes 39. This removes the uncertainty in the isotopeshift measurements induced by the imperfect knowledge of the ion beam energy. Furthermore, the possibility of performing mass measurements or mass separation while ${ }^{335}$ simultaneously performing laser spectroscopy will be studied. It is expected that, besides CLS, also mass separation of rare isotopes will benefit from the enhanced, $30-\mathrm{keV}$ beam energy envisioned in the future setup.

\section{Conclusion}

For the first time, collinear laser spectroscopy has been applied in a MR-ToF device. This new technique, under development within the MIRACLS project at ISOLDE, CERN, offers the potential of improving conventional fluorescencebased CLS significantly by trapping ion bunches for multiple revolutions. While first steps are being taking in a PoP setup at CERN, simulations and preparations are already under way to design a device operating at $30 \mathrm{keV}$.

\section{Acknowledgments}

The research leading to these results has received funding from the European Research Council (ERC) under the European Unions Horizon 2020 research and innovation programme under grant agreement No 679038. P.F. acknowledges Support by the German Ministry for Education and Research (BMBF, 05P15HGCIA). We would like to express our gratitude to $\mathrm{K}$. Blaum for many fruitful discussions and his continuous support to the project. We thank M. Bissell, M. Borge, J. Dilling, R. Garcia Ruiz, M. Kowalska, R. Neugart, G. Neyens, and R. Sanchez, for their help and advice, especially during the beginning of the project. We are grateful to Z. Andjelkovic, T. Murböck, and S. Schmidt for sharing their experience on a compact magnesium ion source as well as to S. Sailer and L. Bartels for their earlier contributions.

\section{References}

[1] K. Blaum, J. Dilling, and W. Nörtershäuser, "Precision atomic physics techniques for nuclear physics with radioactive beams," Physica Scripta, vol. T152, p. 014017, Jan 2013.

[2] P. Campbell, I. Moore, and M. Pearson, "Laser spectroscopy for nuclear structure physics," Progress in Particle and Nuclear Physics, vol. 86, pp. 127 - 180, 2016

[3] R. Neugart et al., "Collinear laser spectroscopy at isolde: new methods and highlights," Journal of Physics G: Nuclear and Particle Physics, vol. 44, no. 6, p. 064002, 2017.

[4] R. Catherall et al., "The isolde facility," Journal of Physics G: Nuclear and Particle Physics, vol. 44, no. 9, p. 094002, 2017.

[5] C. Babcock et al., "Quadrupole moments of odd-a 5363mn: Onset of collectivity towards $\mathrm{n}=40$," Physics Letters B, vol. 760, pp. $387-392,2016$.

[6] M. J. G. Borge and B. Jonson, "Isolde past, present and future," Journal of Physics G: Nuclear and Particle Physics, vol. 44, no. 4, p. 044011, 2017.

[7] H. Wollnik and M. Przewloka, "Time-of-flight mass spectrometers with multiply reflected ion trajectories," International Journal of Mass Spectrometry and Ion Processes, vol. 96, no. 3, pp. $267-274,1990$.

[8] D. Zajfman et al., "Electrostatic bottle for long-time storage of fast ion beams," Phys. Rev. A, vol. 55, pp. R1577-R1580, Mar 1997.

[9] W. H. Benner, "A gated electrostatic ion trap to repetitiously measure the charge and $\mathrm{m} / \mathrm{z}$ of large electrospray ions," Analytical Chemistry, vol. 69, no. 20, pp. 4162-4168, 1997.

[10] W. Plaß et al., "Isobar separation by time-of-flight mass spectrometry for low-energy radioactive ion beam facilities," $\mathrm{Nu}$ clear Instruments and Methods in Physics Research Section B: Beam Interactions with Materials and Atoms, vol. 266, no. 19, pp. $4560-4564,2008$. Proceedings of the XVth International 
Conference on Electromagnetic Isotope Separators and Techniques Related to their Applications.

[11] A. Piechaczek et al., "Development of a high resolution isobar separator for study of exotic decays," Nuclear Instruments and ${ }_{420}$ Methods in Physics Research Section B: Beam Interactions with Materials and Atoms, vol. 266, no. 19, pp. 4510 - 4514, 2008. Proceedings of the XVth International Conference on Electromagnetic Isotope Separators and Techniques Related to their Applications.

[12] P. Schury et al., "Multi-reflection time-of-flight mass spectrograph for short-lived radioactive ions," The European Physical Journal A, vol. 42, p. 343, Oct 2009.

[13] J. D. Alexander et al., "Short pulse laser-induced dissociation of vibrationally cold, trapped molecular ions," Journal of Physics430 B: Atomic, Molecular and Optical Physics, vol. 42, no. 15, p. $154027,2009$.

[14] M. Lange et al., "A cryogenic electrostatic trap for long-time storage of kev ion beams," Review of Scientific Instruments, vol. 81 , no. 5 , p. $055105,2010$.

[15] R. Wolf et al., "On-line separation of short-lived nuclei by a multi-reflection time-of-flight device," Nuclear Instruments and Methods in Physics Research Section A: Accelerators, Spectrometers, Detectors and Associated Equipment, vol. 686, pp. 82 $-90,2012$.

[16] F. Wienholtz et al., "Masses of exotic calcium isotopes pin down nuclear forces," Nature, vol. 498, Jun 2013.

[17] R. N. Wolf et al., "Isoltraps multi-reflection time-of-flight mass separator/spectrometer," International Journal of Mass Spectrometry, vol. 349, pp. 123-133, 2013.

[18] F. Wienholtz et al., "Towards ultrahigh-resolution multireflection time-of-flight mass spectrometry at isoltrap," Physica Scripta, vol. 2015, no. T166, p. 014068, 2015.

[19] T. Dickel et al., "Dynamical time focus shift in multiplereflection time-of-flight mass spectrometers," International Journal of Mass Spectrometry, vol. 412, pp. 1 - 7, 2017.

[20] C. Jesch et al., "The mr-tof-ms isobar separator for the titan facility at triumf," in TCP 2014, pp. 175-184, Springer International Publishing, 2017.

[21] S. Kaufman, "High-resolution laser spectroscopy in fast beams," Optics Communications, vol. 17, no. 3, pp. 309 - 312, 1976.

[22] M. Rosenbusch et al., "Towards systematic investigations of space-charge phenomena in multi-reflection ion traps," AIP Conference Proceedings, vol. 1521, no. 1, pp. 53-62, 2013.

[23] L. Schweikhard, M. Rosenbusch, F. Wienholtz, and R. Wolf, "Isobar separation and precision mass spectrometry of shortlived nuclides with a multi-reflection time-of-flight analyzer," Proceedings of Science (PoS), 2014. Proceedings of the Xth Latin Am. Sym. Nucl. Phys. Appl., Montevideo, Uruguay.

[24] M. Rosenbusch et al., "Delayed bunching for multi-reflection time-of-flight mass separation," AIP Conference Proceedings, vol. 1668 , no. 1, p. 050001, 2015.

[25] F. M. Maier et al., "Simulations of a proof-of-principle experiment for collinear laser spectroscopy with a multi-reflection time-of-flight device," Accepted in Hyperfine interactions, March 2019.

[26] T. Murböck et al., "A compact source for bunches of singly charged atomic ions," Review of Scientific Instruments, vol. 87, no. 4, p. 043302, 2016 .

[27] R. N. Wolf, G. Marx, M. Rosenbusch, and L. Schweikhard, "Static-mirror ion capture and time focusing for electrostatic ion-beam traps and multi-reflection time-of-flight mass analyzers by use of an in-trap potential lift," International Journal of Mass Spectrometry, vol. 313, pp. 8-14, 2012.

[28] M. Rosenbusch et al., "Towards systematic investigations of space-charge phenomena in multi-reflection ion traps," AIP Conference Proceedings, vol. 1521, no. 1, pp. 53-62, 2013.

[29] K. Kreim et al., "Nuclear charge radii of potassium isotopes beyond n=28," Physics Letters B, vol. 731, pp. 97 - 102, 2014.

[30] F. Wienholtz, S. Kreim, M. Rosenbusch, L. Schweikhard, and R. Wolf, "Mass-selective ion ejection from multi-reflection timeof-flight devices via a pulsed in-trap lift," International Journal of Mass Spectrometry, vol. 421, pp. 285 - 293, 2017.

[31] A. Nieminen et al., "On-line ion cooling and bunching for collinear laser spectroscopy," Phys. Rev. Lett., vol. 88, p. 094801, Feb 2002.

[32] E. Mané et al., "An ion cooler-buncher for high-sensitivity collinear laser spectroscopy at isolde," The European Physical Journal A, vol. 42, pp. 503-507, Dec 2009.

[33] D. Manura and D. Dahl, "Simion (r) 8.1 user manual," 2013.

[34] W. Ansbacher, Y. Li, and E. Pinnington, "Precision lifetime measurement for the $3 p$ levels of $\mathrm{mg}$ ii using frequency-doubled laser radiation to excite a fast ion beam," Physics Letters A, vol. 139 , no. 3, pp. 165 - 169, 1989.

[35] F. Wienholtz. Private Communications, 2018.

[36] S. Lechner et al., "Fluorescence detection as a new diagnostics tool for electrostatic ion beam traps," Submitted to Hyperfine interactions, March 2019.

[37] W. Nörtershäuser et al., "Nuclear charge radii of $7,9,10 \mathrm{Be}$ and the one-neutron halo nucleus ${ }^{11}$ Be," Phys. Rev. Lett., vol. 102, p. 062503, Feb 2009.

[38] A. Krieger et al., "Frequency-comb referenced collinear laser spectroscopy of be+ for nuclear structure investigations and many-body qed tests," Applied Physics B, vol. 123, p. 15, Dec 2016.

[39] P. Imgram et al. Phys Rev. A - In print, 2019. 